\title{
L'injure-fable dans Othello : race, sexualité et bestialité
}

The Racial Insult-Tale In Othello: Race, Sexuality and Bestiality

\section{Nora Galland}

\section{(2) OpenEdition \\ 1 Journals}

Édition électronique

URL : http://journals.openedition.org/shakespeare/5611

DOI : 10.4000/shakespeare.5611

ISSN : 2271-6424

\section{Éditeur}

Société Française Shakespeare

\section{Référence électronique}

Nora Galland, «L'injure-fable dans Othello : race, sexualité et bestialité 》, Actes des congrès de la Société française Shakespeare [En ligne], 38 | 2020, mis en ligne le 26 juin 2020, consulté le 06 juillet 2020. URL: http://journals.openedition.org/shakespeare/5611; DOI : https://doi.org/10.4000/shakespeare. 5611

Ce document a été généré automatiquement le 6 juillet 2020.

(C) SFS 


\title{
L'injure-fable dans Othello : race, sexualité et bestialité
}

The Racial Insult-Tale In Othello: Race, Sexuality and Bestiality

\author{
Nora Galland
}

\section{Introduction}

«I look down towards your feet, but that's a

fable ${ }^{1}$."

Othello, V.ii.279

1 Dans cette citation, Othello observe les pieds de Iago en imaginant voir les sabots fendus du diable mais s'arrête dans son élan par cet avertissement à lui-même : «but that's a fable » (V.ii.279). Le mot anglais «fable » est défini par Thomas Elyot comme synonyme d'apologue dans lequel «[...] beastes or trees doo speake ${ }^{2} »$. Ce terme est également connoté comme le signale Randle Cotgrave qui le définit à son tour comme «[...] fib, lie, leasing, false tale, vnlikelie thing reported [... $]^{3} »$. En usant de références animales, Iago donne une forme littéraire à son injure ${ }^{4}$ en s'inspirant du genre de la fable. Ce genre s'est développé en Grèce ancienne au VI ${ }^{\mathrm{e}}$ siècle avant notre ère grâce à Ésope, esclave affranchi, pour qui l'écriture s'est révélée moyen d'émancipation. Dès ses origines, la fable se construit autour de la complexité des formes de pouvoir et des rapports de force entre les forts et les faibles. Par la forme de la fable, Ésope retrouve d'ailleurs une humanité qui lui était niée en raison de son statut d'esclave. Précisément, dans Othello, Iago s'empare lui aussi de la fable mais pour la pervertir : elle n'est plus un moyen d'accéder à l'humanité mais devient une injure sophistiquée pour nier l'humanité de l'autre. Il fait preuve d'ingéniosité littéraire en attaquant Othello à travers ce que j'appelle « l'injure-fable » que je définirai comme une insulte prenant la forme d'un micro-récit reposant sur une interaction entre au moins deux animaux, représentant des personnages humains, et dont la morale doit être déduite. Dans la scène d'exposition, Iago a recours à l'injure-fable à deux reprises et utilise alors les animaux comme références culturelles afin de parler de sexualité ; d'ailleurs les images 
animales émergent ${ }^{5}$ tout au long de la pièce à travers ce qui apparaît comme un réseau métaphorique tissé en particulier par Iago, la vipère (" that viper ", V.ii.278) de la pièce. Ces images animales symbolisent une sexualité débridée et hédoniste, notamment la pratique du cocufiage. Elles évoquent aussi une agressivité démesurée ou, au contraire, une vulnérabilité dont Iago souhaite profiter. Cependant, les deux exemples suivants sont différents des autres dans la mesure où ils prennent une connotation raciale ${ }^{6}$ en contexte :

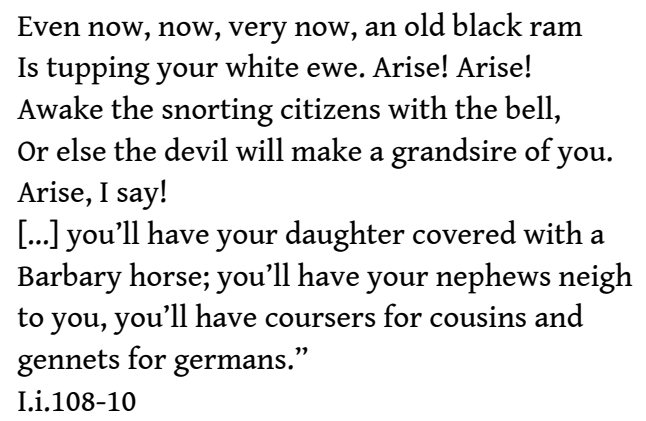

Dans ces injures-fables, Iago nous raconte la relation sexuelle de Desdémone et Othello, censée suivre la cérémonie de mariage qui vient d'avoir lieu, avant le début de la pièce. L'objet de cet article est de proposer une interprétation des injures-fables racistes de Iago à travers la dialectique de la sexualité et de la bestialité. Il s'agira de montrer tout d'abord l'esthétique obscène de l'injure-fable de Iago, qui y a recours afin de représenter de façon crue un acte sexuel choquant; je poursuivrai en soulignant la poétique subversive de l'injure-fable de Iago qui transforme l'animal en bête raciale ; je terminerai par étudier l'herméneutique raciste de l'injure-fable en observant la façon dont les personnages et le public dégagent la morale de l'injure fable.

\section{Esthétique obscène de l'injure-fable : visualiser l'acte sexuel pour choquer}

3 Iago désacralise le mariage de Desdémone et Othello, «[...] an erring barbarian and a super-subtle Venetian [...] » (I.iii.342-3), qui a eu lieu hors scène. Par la crudité de son langage, il sape l'intimité de la nuit de noces qui n'est désormais plus de l'ordre du privé mais portée sur la place publique. Dans l'évocation de l'acte sexuel entre Desdémone et Othello, il ne cherche pas à en suggérer l'érotisme ou la sensualité, ni la pornographie qui pourraient, de façon plus ou moins subtile, éveiller le désir sexuel de son auditoire, Iago ne cherche qu'à en montrer toute l'obscénité, notamment avec l'expression vulgaire : «[...] making the beast with two backs [...]» (I.i.113). Son but est de choquer en évoquant des images sexuelles au moyen de l'hypotypose, aussi appelée « the counterfeit representation " par George Puttenham ${ }^{7}$ qui la définit en ces termes :

The matter and occasion leadeth us many times to describe and set foorth many things, in such sort as it should appeare they were truly before our eyes though they were not present, which to do it requireth cunning: for nothing can be kindly counterfeit or represented in his absence, but by great discretion in the doer.

L'esthétique des images suggérée est indécente: Iago nous fait voir, en relief, l'acte sexuel en train de se faire. Dans les deux injures-fables étudiées dans cet article (citées en introduction), les allitérations en [n], [k], [j], [w] ainsi que les assonances des diphtongues [aI] et [av] créent un effet de résonnance musicale qui renforce l'effet de 
rémanence visuelle engendré par la redondance des deux images. Il comble le manque du hors-scène par cette figure de style en nous permettant de visualiser l'acte obscène.

\section{La vision vulgaire de la copulation entre deux animaux : commentaire sur la sexualité humaine par analogie}

Dans sa première évocation de la nuit de noces, Iago fait référence à un bélier et une brebis, deux animaux de sexe différent appartenant à la même espèce animale. Leur copulation devrait être a priori naturelle mais Iago s'assure de remettre en question la légitimité d'une telle union en ayant recours à des adjectifs qui vont identifier les animaux à Desdémone et à Othello8. Iago procède par analogie et entreprend de construire une correspondance entre la situation de ces deux animaux et celle d'Othello et de Desdémone. La hiérarchie de la Grande Chaîne des Êtres ${ }^{9}$, inspirée d'Aristote, est invoquée implicitement dans cette mise en parallèle des deux animaux et des deux êtres humains. Chaque relation entre ce qui existe dans le monde doit respecter cet ordre hiérarchique pour assurer la continuité du cycle naturel et la survie de tous. Iago s'appuie sur ce sous-entendu culturel de la Grande Chaîne des Êtres pour présenter l'acte sexuel de son injure-fable comme inadmissible et irresponsable. Les critères retenus par Iago pour différencier la brebis et le bélier sont le genre, l'âge et la couleur (noir ou blanc); la couleur, et, dans une moindre mesure, la différence d'âge, empêchent les deux animaux de pouvoir s'accoupler selon la nature. Le bélier et la brebis sont alors racisés par des adjectifs épithètes dont la présence dans la phrase rend le prédicat contre-nature. Jeffrey Masten insiste sur la portée que cette injure-fable a sur le reste de la pièce : "The line economically accomplishes something central in this play : it (re)marks [sic] Othello's blackness, it graphically connects power and sex ; and as many have noted, it makes Othello and Desdemona's connection bestial. $»^{10}$. Cette vision de sexualité animale devient bestiale lorsqu'elle renvoie à deux êtres humains, Othello et Desdémone; elle est aussi raciale par la connotation qu'impliquent les adjectifs épithètes. L'injure-fable a donc pour effet de marquer l'union de Desdémone et d'Othello au sceau de la bestialité interraciale, c'est-à-dire contre-nature.

\section{Le fantasme abject d'un accouplement bestial entre une femme et un animal : la perversion d'un acte zoophile}

6 Après la vision vulgaire d'une copulation animale, Iago va plus loin abordant dans le registre de l'abject avec la suggestion d'un acte zoophile entre une femme et un cheval, qui appartiennent à deux espèces différentes ${ }^{11}$. Dans la copulation animale évoquée précédemment, le bélier est le sujet d'une phrase à la voix active ; alors que dans l'acte sexuel zoophile, la phrase est à la voix passive avec le Barbe introduit comme agent par la préposition «with » à la fin de la proposition. Ce passage de la voix active à la voix passive, qui met l'accent soit sur le thème (sujet) soit sur le rhème (prédicat), montre ainsi le point de vue des deux personnages dans l'évocation de la même scène. Cette manipulation de la syntaxe, passant de l'actif au passif, semble être une stratégie par laquelle Iago nous permet de mieux voir, de façon dynamique, l'acte zoophile. Par la suggestion de cette perversité sexuelle, Iago semble vouloir horrifier Brabantio en le forçant à imaginer l'insoutenable. 


\section{L'ignoble prophétie d'une progéniture monstrueuse ou le cauchemar du patriarche blanc humilié et lésé}

7 Procédant par étape, comme tout bon sophiste, Iago en arrive à la conclusion de son injure-fable, c'est-à-dire aux conséquences de l'union contre-nature entre Desdémone et Othello comme contre-nature. Il prévoit donc l'arrivée d'une progéniture monstrueuse en ayant recours à l'auxiliaire modal «will». Il prédit que Desdémone accouchera non pas d'un hybride mi-animal et mi-humain, mais bien de chevaux. Un tel fantasme n'avait rien d'étonnant à l'époque puisque la descendance d'unions interraciales était souvent décrite comme monstrueuse dans la littérature. Par exemple, Mandeville évoque l'accouplement de noirs avec des femmes babyloniennes, donnant naissance à toutes sortes de monstres :

And that time, the fiends of hell came many times and lay with the women of his generation and engendered on them diverse folk, as monsters and folk disfigured, some without heads, some with great ears, some with one eye, some giants, some with horses' feet and many other diverse shape against kind ${ }^{12}$.

8 Parallèlement, Jean Bodin assure son lecteur que les actes zoophiles sont récurrents en Afrique et ont pour conséquence d'engendrer différents types de monstres : «Promiscuous coition of men and animals took place, wherefore the regions of Africa produce for us many monsters. $»^{13}$. Cependant comme le dit le proverbe, l'Afrique produit toujours quelque chose de nouveau. En effet, avec cette injure-fable, Iago se distingue de la tradition de la paradoxographie, genre regroupant des textes soulignant les caractéristiques improbables et extraordinaires de certains peuples en mêlant description factuelle et merveilleux. Dans son injure-fable qui se passe en réalité au conditionnel, Iago fait accoucher Desdémone d'animaux et non pas d'hybrides ayant des caractéristiques humaines et animales. Ainsi, Iago prend de la distance avec la tradition de la paradoxographie, en imaginant un accouplement entre une femme et un cheval d'une part, et d'autre part, en faisant de leur progéniture, non pas un hybride, mais un pur animal ${ }^{14}$. Un tel scénario est cauchemardesque pour le premier destinataire de l'injure-fable, Brabantio. En effet, l'évocation choquante de l'acte sexuel entre Desdémone et Othello est d'abord une injure dirigée contre lui, qui renvoie à la défaillance de son autorité paternelle ; Thomas Betteridge commente la réaction de Brabantio comme l'expression de deux phénomènes : «[...] coded racism and patriarchal fear $[. ..] »^{15}$. Bien que Desdémone soit directement citée par Iago, c'est son père Brabantio qui s'en trouve gravement insulté et humilié. Iago fait référence à Desdémone en utilisant des pronoms possessifs pour rappeler qu'elle reste sous la responsabilité de son père Brabantio, le patriarche qui a failli à sa responsabilité. L'avenir de sa famille est en jeu dans cette affaire, comme ses multiples appels au clan familial, à sa nation, à sa « race » le montrent bien : «Raise all my kindred » (I.i.163), «Call up my brother !» (I.i.171), «Call up all my people !» (I.i.137), ce qui rejoint les instructions de Iago à Roderigo : «[...] incense her kinsmen [...] (I.i.67); réagissant à l'injure-fable de Iago, Brabantio envisage d'abord un règlement de comptes en famille avant de décider de s'en remettre à la communauté de Venise, « [...] my brothers of the state [...] » (I.ii.96). La faillite de Brabantio et ses conséquences sont soulignées dans l'injure-fable par Iago. C'est une réussite car Brabantio est fou de rage ; la virilité du patriarche est remise en question par cette véritable atteinte à son honneur, et il va demander justice. 


\section{Poétique subversive de l'injure-fable : transformer l'animal en bête raciale}

Brabantio est sous le choc de l'injure-fable qui le rend vulnérable aux procédés de persuasion de Iago. Ce dernier se révèle stratège et sophiste mais surtout poète, prenant le soin de mettre les formes. Nous avons vu que l'injure-fable est adressée directement à Brabantio et vise dans une moindre mesure Desdémone. Néanmoins, sa cible principale reste Othello. Nous avons indiqué que celui-ci était insulté par l'assimilation à des animaux mais il est nécessaire d'analyser cette assimilation qui repose sur l'émergence d'une poétique subversive. Le caractère littéraire de l'injurefable de Iago s'explique par sa qualité de palimpseste dans la mesure où elle s'ancre dans un réseau intertextuel ainsi que par son potentiel de suggestion implicite. L'injure-fable de Iago se caractérise par un renversement sémiotique du littéral en figuré. Le détournement de l'animal en bête raciale est la conséquence de l'effet subversif de la pragmatique de l'injure-fable.

\section{L'étoffement d'une créature hybride dionysiaque en bouc émissaire racial : peindre l'homme noir en hyper-satyre}

10 À travers la métaphore du «old black ram » (I.1.86), Iago relie Othello à la tradition culturelle du mythe du satyre, remontant à l'antiquité grecque. Il s'agit d'une créature sylvestre et hybride, mi-homme mi-bête (ayant des jambes et des oreilles de bélier ou de bouc), que distingue un goût immodéré pour la boisson et la luxure. Cette créature mythologique est régulièrement représentée dans les peintures de la Renaissance comme ithyphallique, errant nue, et cherchant à séduire ou violer des nymphes. Par l'injure-fable, Iago fait d'Othello un satyre plus sauvage et libidineux que les autres; l'adjectif «black » a un rôle d'intensif créant alors une nouvelle catégorie de satyres ou d'hyper-satyres, faisant ainsi de l'homme noir le bouc émissaire du raciste blanc. Iago nourrit ce mythe classique de stéréotypes stigmatisants sur le corps noir masculin remontant à l'antiquité. Dans l'Empire Romain, le corps noir est déjà saturé de connotations péjoratives le faisant apparaître comme en proie aux passions, déserté par la froide raison, et sa laideur physique révèle la laideur morale. Par exemple, l'association de la peau noire à une certaine concupiscence apparaît dans une mosaïque romaine datant du $\mathrm{II}^{\mathrm{e}}$ siècle avant notre ère et se trouvant à l'entrée de thermes à Timgad, en Algérie ${ }^{16}$. Sur cette représentation, on voit un homme noir portant une pelle pour jeter du charbon dans la fournaise afin de maintenir la chaleur des lieux. Il ne porte aucun vêtement et est pourvu d'un énorme pénis d'où s'écoule sa semence. Cette mosaïque présente d'une part l'homme noir comme hypersexualisé et particulièrement fertile, et d'autre part elle fait le lien entre chaleur, charbon et sexualité, un lien que l'on retrouve dans Othello. Roderigo fait référence au «sooty bosom » (I.i.70) d'Othello, reliant la suie à la couleur de sa peau. Othello est bien peint comme un fornicateur ; d'ailleurs l'étymologie du mot reprend l'association de la chaleur et de la sexualité : " [...] lechery as well as a space that is « arched, vaulted like an oven or furnace [...] $»^{17}$ (OED, Étymologie de «fornicate»). La dialectique reposant sur la tension entre l'apollonien et le dionysiaque rejoint la tradition manichéenne du noir à la sexualité malsaine et déviante, et du blanc à la sexualité saine et normale. Iago procède à une sorte de greffe poétique en reliant les deux traditions. Le satyre, mi-homme mi-bélier, 
est racisé; sa peau noire devient la cause et l'effet de son hypersexualisation. Le contexte de l'injure donne une coloration raciale au satyre qui donne de son côté une justification classique et mythologique aux stéréotypes racistes déjà existants sur les hommes noirs ${ }^{18}$. On constate cependant un effet d'étoffement sémiotique faisant d'Othello un hyper-satyre plus sexualisé que les autres.

\section{L'inversion axiologique des métaphores équines : de la noblesse à la bassesse du cheval}

11 Dans l'injure-fable, Iago façonne ses métaphores en utilisant plusieurs races de chevaux qui étaient bien connues des Élisabéthains : "a Barbary horse » (I.i.109) et " coursers » (I.i.110). Ce qui surprend est qu'il profère une injure en ayant recours à des symboles culturels loués pour leur noblesse tout au long de la Renaissance en Europe. En poète subversif, Iago entreprend une inversion axiologique des métaphores équines, faisant de symboles de noblesse à des marques de bassesse. Le croisement était une pratique courante dans l'élevage de chevaux à la Renaissance, pour créer des races de chevaux convenant le mieux à l'usage qui en serait fait. Henry VIII fut le premier des Tudors à investir dans le croisement pour améliorer les races de chevaux anglais: "Courtiers and members of the royal household were commissioned to buy mares for breeding purposes in northern and southern Europe and the Ottoman Empire throughout Henry's reign [...] $»^{19}$. Les chevaux napolitains (« coursers » (1.1.110) ou Napoletano en anglais) étaient très prisés, en Europe aux XVI ${ }^{e}$ et XVII siècles, d'abord pour la guerre puis pour les parades de Haute École à la cour. Cette race de chevaux a été mise en point au XVe siècle grâce à un croisement avec des chevaux venant de l'Empire Ottoman. Le Barbe est aussi très connu à l'époque et jouit d'une très bonne réputation autant comme cheval de guerre que comme cheval de selle. Le Barbe et le cheval Napolitain sont des signifiants culturels dotés d'une connotation positive, révélant la noblesse, le pouvoir et l'autorité du propriétaire. Cette connotation est pervertie par Iago qui inverse la valeur axiologique de ces animaux en en faisant des symboles de stigmatisation négative. Dans le contexte de la poétique subversive de l'injure-fable, Iago fait du Barbe la métaphore péjorative stigmatisant Othello comme bête muette et lubrique, et du cheval Napolitain la métaphore négative incarnant la progéniture monstrueuse engendrée par l'union contre-nature de Desdémone et d'Othello.

\section{Le genet espagnol comme métonymie du métissage post- Reconquista : le contexte de la pureté de sang et de la dégénérescence raciale dans l'Espagne de la première modernité}

12 Une autre race de cheval est utilisée comme métaphore par Iago pour connoter la descendance de Desdémone et d'Othello dans le cadre de son injure-fable : "gennets » (I.i.110). Il s'agit du genet qui n'apparaît qu'une seule fois dans tout le corpus dramatique shakespearien. Cette race de chevaux est définie comme «1. A small Spanish horse » dans l'OED mais une telle définition lisse une complexité qui fait toute la richesse de l'Espagne qui s'est construite sous les dominations chrétienne et musulmane qui se sont succédé de 711 à la Reconquista de 1492 par Ferdinand et Isabelle. Le genet est bien plus qu'une simple métonymie de l'Espagne ${ }^{20}$. C'est un cheval déjà utilisé par les tribus Berbères des Zénètes, connus pour leurs excellents cavaliers, 
lorsque les Arabes arrivent en Afrique du Nord ${ }^{21}$. Quelques années plus tard, ils poursuivent leur conquête en Espagne et y introduisent le genet. Ce cheval va rester un signifiant symbolique important dans les traditions culturelles de l'Al-Andalus. Après les Berbères, ce sont les Maures qui se sont approprié le genet puis les Espagnols. Après la Reconquista, un tribunal de l'inquisition se met en place pour distinguer les Vieux Chrétiens et les Nouveaux Chrétiens, c'est-à-dire les musulmans et les juifs nouvellement convertis au Christianisme. Ces derniers avaient eu le choix entre la conversion ou l'exil vers l'Afrique du Nord. Cette alternative avait été décidée à l'époque en raison de l'émergence de l'idéologie de la pureté de sang selon laquelle les Vrais Chrétiens avaient un sang pur, contrairement aux Nouveaux Chrétiens, que même la conversion religieuse ne pouvait pas à les débarrasser de leur impureté. Les statuts de sang servaient donc à discriminer les purs des impurs. Or, le genet est le symbole du métissage de l'Espagne qui s'est construite grâce à plusieurs héritages culturels ${ }^{22}$. Cependant, cette hybridité était perçue comme un exemple de dégénérescence raciale par le tribunal de l'Inquisition espagnole (1471-1561) estimant la politique de pureté de sang comme nécessaire ${ }^{23}$. Le genet représente le métissage dans la mesure où il était au cœur des spectacles de cannes (juegos de cañas), qui étaient des exhibitions de sport équestre dans lesquelles des centaines de cavaliers rejouaient les batailles entre chrétiens et musulmans ${ }^{24}$. Ces derniers étaient finalement vaincus et finissaient désarçonnés avant de se convertir au christianisme. Sous la poétique subversive de Iago, le genet perd sa connotation méliorative et devient la métonymie de la dégénérescence raciale qui a justifié la politique de la pureté de sang. L'animal renvoie à une vision négative du métissage : c'est la dégradation d'une pureté naturelle et l'aliénation d'une identité nationale par une occupation étrangère. Dans l'injurefable de Iago, le genet est donc un animal dont la symbolique politique met en garde contre le métissage qui s'attaque à la stabilité et à la pureté de la nation, comme tout ce qui vient des Maures.

\section{Herméneutique raciste de l'injure-fable : interpréter la morale de l'allégorie}

13 La morale raciste de l'injure-fable doit être déduite car elle n'est pas explicitement donnée par Iago. Le premier destinataire, Brabantio, l'interprète et s'explique. D'autres personnages adoptent la même démarche herméneutique en partageant leur réflexion sur le message didactique de l'injure-fable. Ainsi Iago apparait comme une sorte de maïeuticien faisant accoucher Brabantio d'un discours raciste. L'injure-fable a donc des effets sur les personnages dans la mesure où sa réception n'est pas consensuelle mais elle a aussi une fonction dramatique en ayant un effet précis sur l'intrigue de la pièce.

\section{La fonction entropique de l'injure-fable : lago comme chœur d'une action contrefactuelle}

Dans cette scène d'exposition, il n'y a pas d'action directe mais de la narration. Iago joue le rôle d'un conteur se produisant devant un auditoire plutôt que devant des spectateurs. L'acte sexuel dont parle l'injure-fable est raconté comme ayant lieu au moment de l'énonciation par Iago qui emploie le présent continu. D'autre part, la répétition du marqueur déictique «now » (I.i.86), intensifié par l'adverbe « very» (I.i. 
86), indique que l'action est bien en cours. On apprend plus tard que Iago extrapole lorsqu'il émet l'injure-fable car l'acte sexuel en question n'est pas en train de se produire au moment où il parle; d'ailleurs le mariage de Desdémone et Othello n'est jamais consommé de façon certaine. Il reste en suspens tout au long de la pièce jusqu'à la mort du couple. La consommation du mariage, qui lui aurait apporté une certaine légitimité aux yeux de la communauté, n'a pas lieu et l'acte sexuel narré par Iago n'est pas accompli. Iago est donc le chœur d'une action contrefactuelle. En personnage démiurge, Iago crée une action dramatique ex nihilo; il fait se produire une conséquence sans cause car ce qui pousse Brabantio à réagir n'a en réalité jamais eu lieu. Cette scène d'exposition semble quelque peu atypique car aucune réelle action ne se produit sur scène ; il n'y a que Iago faisant naître une tension dramatique à partir de rien. L'injure-fable est ainsi utilisée par Iago comme catalyseur de chaos et son fonctionnement est symptomatique de la structure d'une pièce dont l'action dramatique semble ne reposer que sur du rien.

\section{La surenchère fabuleuse de Roderigo et Brabantio : le travail de lago, la muse raciste}

L'injure-fable a un effet de surenchère fabuleuse chez Roderigo et Brabantio qui, tour à tour, donnent leur interprétation et la morale qu'ils en ont tirée. Roderigo surenchérit après le discours de Iago pour persuader Brabantio de la véracité des faits relatés. Après Iago, jouant ici le rôle de muse raciste, Roderigo rappelle la fugue de Desdémone en précisant qu'elle a rejoint Othello. Reprenant brièvement la thématique bestiale de l'injure-fable de Iago en désignant Othello par «the gross clasps of the lascivious moor » (I.i.122), Roderigo donne sa morale de l'allégorie de Iago, envers la leçon à tirer de l'injure-fable; Desdémone a transgressé son devoir d'obéissance à son père et et oublié sa responsabilité envers sa communauté : «Your daughter [...] hath made a gross revolt,/ Tying her duty, beauty, wit, and fortunes/ In an extravagant and wheeling stranger [...] » (I.i.129-132). Roderigo insiste sur la portée politique de la rébellion de Desdémone alors que l'injure-fable de Iago reposait avant tout sur son immoralité. La surenchère de Roderigo grossit encore l'injure-fable de Iago et parvient à convaincre Brabantio de l'urgence de la situation. En effet, à la suite de l'interprétation de Roderigo, Brabantio se met en mouvement, adhérant alors à la morale raciste de l'injure-fable délivrée par Roderigo, comme il le dit lui-même: "It is too true an evil [...]» (I.i.156). Dans la scène suivante, lorsque Brabantio fait face à Othello, il l'accuse même d'avoir usé de sorcellerie pour envoûter sa fille : «Judge me the world if 'tis not gross in sense/ That thou hast practiced on her with foul charms,/ Abused her delicate youth with drugs or minerals/ That waken motion.» (I.iii.72-5). On remarque un nouveau cas de surenchère fabuleuse chez Brabantio qui procède comme Iago, en créant à partir de rien, du moins rien d'autre que son intime conviction ou précisément ses préjugés racistes. Il crée de toutes pièces un scénario dans lequel Othello est le seul responsable, où sa fille est une victime qu'il faut sauver d'un enchantement. Encore dans le déni, Brabantio interprète l'injure-fable de Iago en distinguant racialement les Vénitiens des Maures; pour lui, seul le surnaturel peut expliquer une union aussi contre-nature : «[...] a maid, so tender, fair, and happy/ So opposite to marriage that she shunned/ The wealthy curled darling of our nation, / Would ever have [...] such a thing as thou. » (I.iii.66-71). 


\section{La dynamique éristique de l'injure-fable de lago ou le procès d'Othello : le rôle du jugement officiel}

S'estimant véritablement injurié, Brabantio souhaite entamer des poursuites judiciaires afin de demander réparation et de faire annuler le mariage entre sa fille et Othello. Brabantio s'attend à un jugement favorable car il est de la même race que les Vénitiens contrairement à Othello. Dès que Brabantio annonce à Lodovico que sa fille a été corrompue par de la magie, celui-ci se déclare prêt à utiliser " the bloody book of law " (I.ii.67) pour lui rendre justice. Néanmoins, en raison du danger d'invasion imminent par les Turcs, Lodovico doit rester pragmatique et choisit de ne pas retenir « the vices of [his] blood» (I.iii.124) comme charges contre Othello. Les témoignages d'Othello et de Desdémone remettent en question le bien-fondé de l'injure-fable. Othello fait même preuve d'éloquence et d'humilité lorsqu'il se défend d'avoir trompé Desdémone. D'autre part, il n'apparaît pas du tout comme dominateur puisqu'il demande de laisser la parole à Desdémone afin qu'elle s'exprime elle-même. Celle-ci le fait en réitérant son consentement dans cette union. Ils sont tous les deux très différents de ce qui était d'abord suggéré par l'injure-fable dans la scène d'exposition: Othello n'est pas l'hypersatyre muet et bestial «montant » Desdémone, passive et silencieuse telle une jument résignée. Le contraste entre les points de vue de Brabantio et Roderigo d'une part, et ceux de Desdémone et Othello d'autre part, fait émerger une dynamique éristique qui ébranle la véracité de l'injure-fable de Iago. L'écart entre res et verba apparaît ici de façon évidente-ce qu'a dit Iago dans la scène 1 est remis en question par ce qui se passe dans la scène 3. L'injure-fable est remise en question par le jugement final de Lodovico déclarant que les faits rapportés ne constituent pas réellement une injure pour Brabantio, c'est-à-dire une atteinte à ses droits de patriarche blanc : "Your son in law is far more fair than black. » (I.iii.287). Brabantio se résigne à abandonner sa fille à la fin du procès. Il reste au public de décider si Othello est coupable ou non, et d'adhérer à la morale raciste de l'injure-fable ou non. Othello lui-même fait référence à cette morale raciste en soupirant : " And yet, how nature erring from itself, - » (III.iii. 226), reprenant ainsi les mots exacts de Brabantio prononcés au début de la pièce, en parlant de sa fille : «[...] err / Against all rules of nature » (I.iii.100-1). Iago s'empresse d'ailleurs de confirmer l'incohérence d'une telle union qui va contre les lois de la logique et de la nature, rappelant ce que Desdémone a décliné pour choisir Othello: « [...] many proposed matches / Of her own clime, complexion, and degree, / Whereto we see in all things nature tends-» (III.iii.228-30). Malgré le jugement du procès à la fin du premier acte, la morale raciste de l'injure-fable continue de hanter la pièce et de tenter le spectateur/lecteur.

\section{Conclusion}

17 La référence aux animaux est un procédé de distanciation que Iago utilise pour poser un jugement moral sur une action humaine. À travers l'injure-fable, Iago cherche d'abord à choquer en évoquant une image vulgaire de copulation animale perverse puis en suggérant un acte zoophile entre un cheval et une femme. Ces images sexuelles montrent la bestialité du rapport sexuel imaginé entre Desdémone et Othello. Cette sexualité est dénoncée comme anormale et immorale comme une menace pour la lignée de Brabantio et un danger pour la communauté vénitienne dans son ensemble. 

l'action décrite mais aussi par la charge symbolique des animaux évoqués. Iago façonne son injure-fable à la façon d'un palimpseste en la reliant à plusieurs traditions littéraires et culturelles. Il fait montre de ses qualités de poète en pervertissant la dimension sémiotique des animaux présents dans l'injure-fable. La langue de Iago inverse le bien en mal : les références animales sont mises sens dessus-dessous et marquée du sceau pervers de Iago qui les détourne de leur symbolique originale. L'injure-fable marque les esprits et demande à être interprétée.

Il est intéressant d'étudier les différentes traductions en français des passages analysés et de nous concentrer sur la façon dont les traducteurs utilisent les procédés d'adaptation et d'équivalence culturelle pour parler de la race. Certains effacent toute connotation raciale comme Pierre-Antoine de la Place qui traduit « the old black ram is tupping your white ewe» par «le loup est dans la bergerie» en $1746^{25}$ alors que d'autres la gardent comme François-Victor Hugo en $1860^{26}$ ou François Guizot en $1873^{27}$. Des variations apparaissent également sur le choix des animaux, certains traducteurs font le choix d'introduire de nouveaux animaux comme Jean Aicard qui remplace le vieux bélier noir par «un loup noir » et le genet en "pur-sang arabe » en $1899^{28}$. La traduction de l'injure-fable est donc un véritable défi pour le traducteur qui doit s'assurer de traduire l'injure-fable en conservant son potentiel implicite afin de tenter le spectateur/lecteur libre d'en tirer une morale raciste, comme cela se fait dans le texte-source anglais.

\section{NOTES}

1. L'édition utilisée comme référence à Othello tout au long de l'article est la suivante: William, Shakespeare, The Norton Shakespeare : Digital Edition (Troisième Édition), Stephen Greenblatt, Walter Cohen, Suzanne Grosset, Jean E. Howard, Katharine Eisamen Maus, Gordon McMullan (éds.), New-York: W. W. Norton \& Company, 2016.

2. Entrée «Apologus » dans Thomas Elyot, The Dictionary of Sir Thomas Elyot, Londres, Thomas Berthelet, 1538, STC ( $2^{e}$ éd.) / 7659, consulté (via EEBO) le 11 novembre 2018.

3. Entrée « Fable : $\mathrm{f}$. d dans Randle Cotgrave, A Dictionary of the French and English Tongues, Londres, Adam Islip, 1611, STC ( $2^{e}$ éd.) 5830, consulté (via EEBO) le 10 novembre 2018.

4. Voir Dominique Lagorgette, «Termes d'adresse, acte perlocutoire et insultes: la violence verbale dans quelques textes des $14^{\mathrm{e}}, 15^{\mathrm{e}}$ et $16^{\mathrm{e}}$ siècles", SENEFIANCE, 36, 1994, p. 317-332; Dominique Lagorgette et Pierre Larrivée, «Les insultes: approches sémantiques et pragmatiques », Langue Française, 144, 2004 ; Evelyne Larguèche, L'Effet-injure: De la pragmatique à la psychanalyse, Paris, Presses Universitaires de France, 1983 ; Evelyne Larguèche, L’Injure à fleur de peau, Paris, L'Harmattan,1993; Nathalie Vienne-Guerrin, Shakespeare's Insults: A Pragmatic Dictionary, Londres, Bloomsbury, 2016.

5. Il est à noter que l'injure-fable implique une interaction entre au moins deux animaux. La référence qu'Othello fait au chien circoncis pour s'injurier lui-même n'est donc pas une injurefable. Ici, la métaphore du chien est statique, alors que les animaux de l'injure-fable sont en 
mouvement et au cœur d'une dynamique. Dans ce cas précis, on parlera d'injure allégorique et non d'injure-fable.

6. Cet article adopte une approche post-coloniale de la race telle qu'elle est définie par la théorie critique de la race. En conséquence, la race n'est pas considérée comme le monopole de l'époque moderne, période à laquelle émerge des théories pseudo-scientifiques pour légitimer une théorie des races humaines. Sur le caractère protéen du concept de race, voir Étienne Balibar et Immanuel Wallerstein (éds.), Race, Nation, Classe: Les identités ambiguës, Paris, Editions de La Découverte, 1988; Albert Memmi, Le racisme, Paris, Gallimard, 1982; Magali Bessone, Sans distinction de race : une analyse critique du concept de race et de ses effets pratiques, Paris, Vrin, 2013 ; Hourya Bentouhami-Molino, Race, culture, identités. Une approche féministe et postcoloniale, Paris, Presses Universitaires de France, 2015. Sur le champ de recherche (encore bien trop marginalisé en France) des études de la race dans la première modernité, voir, sans prétention d'exhaustivité, Imtiaz Habib, Shakespeare and Race: Praxis in the Early Modern Period, New-York, University Press of America, 1999 ; Ania Loomba, Gender, Race, Renaissance Drama, Manchester, Manchester University Press, 1989 ; Kim F. Hall, Things of Darkness : Economies of Race and Gender in Early Modern England, Ithac, New-York, Cornell University Press, 1995 ; Mary Floyd-Wilson, English Ethnicity and Race in Early Modern Drama, Cambridge, Cambridge University Press, 2003 ; Sujata Iyengar, Shades of Difference: Mythologies of Skin Color in Early Modern England, Philadelphie, University of Pennsylvania Press, 2005 ; Imtiaz Habib, Black Lives in the English Archives, 1500-1677: Imprints of the Invisible, Burlington, Ashgate, 2008 ; Nabil Matar, Gerald MacLean (éds.), Britain and the Islamic World, 1558-1713, Oxford, Oxford University Press, 2011 ; Matthieu Chapman, Anti-Black Racism in Early Modern English Drama: The Other 'Other', New-York, Routledge, 2016; Patricia Akhimie, Shakespeare and the Cultivation of Difference: Race and Conduct in the Early Modern World, New-York, Routledge, 2018.

7. George Puttenham, The Arte of English poesie. Contriued into three bookes: the first of poets and poesie, the second of proportion, the third of ornament, Londres, Richard Field, 1589, STC ( $2^{\mathrm{e}}$ éd.) / 20519, consulté (via EEBO) le 10 novembre 2018.

8. Voir Andrew William Barnes, Post-closet Masculinities in Early Modern England, Lewisburg: Bucknell University Press, 2009, p. 99.

9. Voir Gabriel Egan, «Gaia and the Great Chain of Being » dans Dan Brayton, Lynne Bruckner (éds.), Ecocritical Shakespeare, Burlington, Ashgate, 2011, p. 57-70.

10. Jeffrey Masten, «Chapter 8: All Is Not Glossed: Editing Sex, Race, Gender, and Affect in Shakespeare " dans Queer Philologies: Sex, Language, and Affect in Shakespeare's Time, Philadelphie, University of Pennsylvania Press, 2016.

11. Sur l'utilisation de métaphores animales, des distinctions et catégories entre animaux, mais aussi des interactions entre animaux de différentes espèces, voir Laurie Shannon, The Accommodated Animal : Cosmopolity in Shakespearean Locales, Chicago, University of Chicago Press, 2013.

12. John Mandeville, The voyages and trauailes of Sir Iohn Mandeuile Knight Wherein is set downe the way to the Holy Land, and to Hierusalem: as also to the lands of the great Caane, and of Praester Iohn, Inde, and diuers other countries: together with the many and strange meruailes therein, Londres, Thomas Snodham, 1625, STC (2e éd.) / 17253, consulté (via EEBO) le 10 novembre 2018.

13. Jean Bodin, "Chapter 5: The Correct Evaluation of Histories", Method for the Easy Comprehension of History, trans. Beatrice Reynolds, New-York, Columbia University Press, 1945, p. 105

14. Sur la paradoxographie, voir Anton Westermann, Paradoxographoi, Scriptores rerum mirabilium Graeci, Brunswick, 1898 ; Laurent Gourmelen, «Est-ce un homme, est-ce un animal ? Peuples hybrides aux marges de l'humanité : l'exemple des Cynocéphales » dans Arlette Bouloumié (éd.), Particularités physiques et Marginalité dans la Littérature, Recherche sur l'imaginaire, Cahier XXXI, Presses Universitaires d'Angers, 2005, p. 17-35. 
15. Thomas Bettteridge, "The Redundancy of Language in Othello", Shakespearean Fantasy and Politics, Hatfield, University of Hertfordshire Press, 2005, p. 133-161, p. 145.

16. Voir Ania Loomba, "Religion, Colour, and Racial Difference" dans Shakespeare, Race, and Colonialism, Oxford, Oxford University Press, 2002, p. 45-74, p. 50.

17. Entrée «Fornicate, etymology » dans The Oxford English Dictionary, $2^{\mathrm{e}}$ éd., Oxford, Oxford University Press,

18. Cette connotation ne va pourtant pas de soi. Dans l'OED, l'un des sens du nom « Ram » est « 1.c. colloq. A lecherous or sexually voracious man; (also) a sexually aggressive or domineering man.». Aucune nuance n'est apportée pour introduire la connotation raciale de l'occurrence présente dans Othello qui est le premier exemple cité. Seule la connotation sexuelle est mise en avant mais la connotation raciale est ignorée. Pourtant, elle est fondamentale dans la mesure où elle est le cœur d'une injure qui attaque Othello et en fait un bouc émissaire racial.

19. Peter Edwards et Graham Elspeth (éds.), "Introduction: The Horse as Cultural Icon: The Real and the Symbolic Horse in the Early Modern World" dans The Horse as Cultural Icon: The Real and the Symbolic Horse in the Early Modern World, Leiden, Koninklijke Brill NV, 2012, p. 1-36, p. 5.

20. Voir Barbara Fuchs, "Chapter 4: Playing the Moor", Exotic Nation: Maurophilia and the Construction of Early Modern Spain, Philadelphia: University of Pennsylvania Press, 2009, p. 88-115, et plus particulièrement p. 89-94 sur le genet.

21. Selon l'entrée « Jennet, etymology » de l'OED, ce mot vient du Berbère : « Zenāta [meaning] 'a great Berber nation noted for the valour of its cavalry'».

22. Voir Ken Parker, "Chapter Five: Reading 'Barbary' in Early Modern England 1550-1685 », dans Matthew Birchwood et Matthew Dimmock (éds.), Cultural Encounters Between East and West, 1453-1699, Newcastle-Upon-Tyne, Cambridge Scholars Press, 2005, p.77-106; Virginia Mason Vaughan, "Chapter 3: Representing the King of Morocco» dans Gitanjali Shahani \& Brinda Charry (éds.), Emissaries in Early Modern Literature and Culture: Mediation, Transmission, Traffic 1550-1700, New-York, Routledge, 2009, p. 77-94.

23. Voir Béatrice Pérez, "Une institution dans la genèse d'un État moderne ", dans Raphaël Carrasco (éd.), Aux Premiers temps de l'Inquisition espagnole (1478-1561), Montpellier, Presses universitaires de la Méditerranée, 2002, p. 9-49.

24. Voir Karen Raber, "A Horse of a Different Color: Nation and Race in Early Modern Horsemanship Treatises", dans Karen Raber et Treva J. Tucker (éds.), The Culture of the Horse: Status, Discipline, and Identity in the Early Modern World, New-York, Palgrave Macmilan, 2005, p. 225-43.

25. Pierre-Antoine de La Place (éd. et trad.), Le Théâtre anglois, Londres, 1746-1749.

26. William Shakespeare, Euvres Complètes, François-Victor Hugo (trans.), Paris, Pagnerre, 1860.

27. William Shakespeare, Euvres Complètes, François Guizot (trans.), Paris, Librairie Académique Didier, 1873.

28. William Shakespeare, Othello, Le More de Venise, Jean Aicard (trans.), Paris, Ernest Flammarion, 1899.

\section{RÉSUMÉS}

Dans Othello, certains personnages ont recours aux animaux pour évoquer une libido hors-norme, la défaite de la raison écrasée par les pulsions sexuelles mais aussi des pratiques sexuelles 
extrêmes. Si les animaux sont associés à la sexualité à travers une série de métaphores et de comparaisons tout au long de la pièce, ils sont particulièrement utilisés pour symboliser la luxure, une sexualité répréhensible et atypique. Ainsi la peur du métissage est exploitée par Iago lorsqu'il annonce à Brabantio que sa fille Desdémone est en train de commettre un acte contrenature en couchant avec Othello, aussi contre-nature que si elle couchait avec un "bélier noir » ou même « un Barbe ». Ces références animales ne se contentent pas de symboliser simplement la luxure : dans ce contexte précis, ces animaux sont au cœur d'injures racistes. Ces exemples sont importants dans la mesure où ils illustrent ce que j'appelle « l'injure-fable raciste ». Cet article se propose de faire comprendre comment l'injure-fable entremêle race, sexualité et bestialité à travers l'acte injurieux.

In Othello, some characters resort to animals to evoke a disproportionate libido overcoming reason as well as extreme sexual practice. If animals are linked to sexuality through a series of metaphors and comparisons in the play, they often stand for lust-a reprehensible, unconventional type of sexuality. So, the fear of miscegenation is clearly alluded to when Iago announces to Brabantio that his daughter Desdemona is committing an unnatural act by having sex with Othello, as unnatural as if she had sex with "a black ram" or even "a Barbary horse". More than mere symbols of lustful sexuality, these animal references are here racial-in this context, the animals become the core of racial insults. These examples are significant insofar as it is a case in point of what I am calling "racial insult-tale". In this paper, the issue at stake will be to understand how race, sexuality, and bestiality are intermingled through the insulting act.

\section{INDEX}

Keywords : Othello, race, sex/sexuality, insult, black ram, Barbary horse, animal studies, gender, masculinity, early modern race studies

Mots-clés : Othello, race, sexe/sexualité, insulte, black ram, Barbary horse, animal studies, genre, masculinité, early modern race studies

\section{AUTEUR}

\section{NORA GALLAND}

IRCL UMR 5186 CNRS, Université Paul Valéry-Montpellier 3 